\title{
Bacteriological Profile of Blood Stream Infections Along with their Antibiogram at Government Cancer Hospital, Aurangabad
}

\author{
Mukta Sawargaonkar*, Nazneen Siddiqui, Joson Mathew and Arvind Gaikwad \\ Department of Microbiology, Govt Cancer Hospital and Research Centre, \\ Aurangabad, Maharashtra, India \\ *Corresponding author
}

\begin{abstract}
A B S T R A C T
Keywords

Blood stream infections, Gram negative, Gram

Positive, MRSA, MDRO

Article Info

Accepted:

17 April 2019

Available Online:

10 May 2019

Bacterial bloodstream infections are important causes of morbidity and mortality globally. The aim of present study was to determine the bacterial profile of bloodstream infections and their antibiotic susceptibility pattern among the clinically diagnosed cases of sepsis in cancer patients. In the present study, etiological and antimicrobial susceptibility profile of blood cultures over a period of 1 year at a tertiary cancer care hospital was done. Blood culture positive isolates were identified using standard microbiological methods and by Manual method. The antibiotic susceptibility pattern of the organisms was performed by Kirby-Bauer disc diffusion method. There were 242 blood culture samples, of which 97 (40\%) were identified to be culture positive. Out of 97 positive cultures, Gram positive were $60(62 \%)$ and Gram negative were 37 (38\%). The most common Gram-positive isolate was MRSA (38\%) and Gram-negative isolate was Escherichia coli (35\%). Escherichia coli showed highest sensitivity to colistin (100\%) and sensitivity to meropenem and Polymyxin B was $92 \%$ each. High degree of resistance was found to cephalosporins and piperacillin + tazobactam. The results indicate high level of antimicrobial resistance among Gram negative bacilli in septicemic patients. The results warrant continuous monitoring of antimicrobial pattern so as to build geographical epidemiological data.
\end{abstract}

\section{Introduction}

Blood stream infections are an important cause of mortality and morbidity and are amongst the most common health care associated infections (1).

Infectious complications consequent to the immunosuppressive therapy has become a major cause of morbidity and mortality in cancer patients (2).
Blood stream infections increase the length of hospital stay, cause significant morbidity and mortality and increase the cost of stay. Situation further deteriorates with increasing rate of multidrug resistance. The crude mortality rate due to BSIs in cancer patients ranges from $18 \%$ to $42 \%$ (3-6).

The organisms and their antibiotic susceptibility pattern vary among different healthcare facilities and geographical areas. Blood culture is the single most reliable 
procedure for bacterial isolation and detection. The aim of the present study was to determine the bacterial profile of bloodstream infections and to assess the antibiotic susceptibility pattern of the major pathogens among the clinically diagnosed cases of sepsis in cancer patients.

\section{Materials and Methods}

This was a retrospective study conducted at a tertiary care hospital for cancer patients. We analyzed all blood samples sent for bacterial culture during the year 2018

A total of 242blood samples from clinically suspected cases of sepsis, received in the microbiology laboratory of 100-bedded cancer hospital over duration of one year, were included in the study. Blood samples were collected before the administration of antibiotics. Relevant details of the patients were recorded. Blood was taken in BHI Broth 1:5 ratio with all aseptic precaution. The blood culture bottles were kept in incubator for 12-18 hrs then sub cultures were done on blood agar, MacConkey agar. The growth obtained was identified by colony morphology, Gram stain of the isolated colonies, standard microbiological, and biochemical test.

The antibiotic susceptibility pattern of the isolated organisms was performed by KirbyBauer disc diffusion method on MuellerHinton agar plates and results were interpreted as per the Clinical and Laboratory Standards Institute (CLSI) 2016 guidelines. Cefoxitin disc diffusion method was used to identify MRSA (Methicillin resistant Staphylococcus aureus) as per CLSI guidelines. MDR (Multi drug resistant) was defined as non-susceptibility to at least one agent in three or more antimicrobial categories
The antibiotic discs that were used to identify the susceptibility pattern of the gram-negative pathogens and their concentrations include amikacin (30 mcg), amoxicillin+clavulanic acid (20/10 mcg), ceftazidime (30 mcg), ceftriaxone $(30 \mathrm{mcg})$, cefepime $(30 \mathrm{mcg})$, cefoperazone + sulbactam $(75 / 30 \mathrm{mcg})$, meropenem $(10 \mathrm{mcg})$, piperacillin + tazobactum (100/10 mcg), levofloxacin (5 mcg), cotrimoxazole (1.25/23.75).

\section{Results and Discussion}

This study was carried out from January 2018 -December 2018 with 242 blood samples received from patients suspected of having blood stream infections attending and admitted in Government cancer Hospital Aurangabad, Maharashtra. Relevant details viz. medical registration number, laboratory number, age and sex of the patients were recorded. Culture positivity was seen in 97 (40.08\%) samples and 145 (59.92\%) samples were sterile.

Out of 97(40.08\%) positive cultures, $60(61.86 \%)$ showed gram positive and $30(38.14 \%)$ were gram negative

Total number of samples- 242

Total Number of Isolates- $97 / 242(40.08 \%)$

Sterile Samples - 145/242 (59.92\%)

Gram Positive-60/97 (61.86\%)

MRSA - 23/60 (38.33\%)

MSSA - $19 / 60(31.67 \%)$

CONS - 16/60 (26.67\%)

ENTEROCOCCUS - 2/60(3.33\%)

Gram Negative - 37/97 (38.14\%)

E.coli-13/37 (35.14\%)

Pseudomonas - 7/37 (18.92\%) 
NON-FERMENTERS - 7/37 (18.92\%)

Acinetobacter - 6/37 (16.21\%)

Kleibsiella - 4/37 (10.81\%)

This study gives information on the distribution of bacterial isolates causing blood stream infections with their antibiotic susceptibility pattern which plays an important role in effective management of patients in septicemia. Our study has shown blood culture positivity rate to be $40.01 \%$ which was approximately similar to Nikita Vasudeva et al., (7) which showed $31.2 \%$, Wasihun et al., (8) showed 28\%, Radha Rani, et al., (9) showed $27.16 \%$. This is in contrast to other studies which have shown positive rates ranging between $9.94 \%-11.2 \%$. (1014). Such differences in positivity rates may be due to the difference in methodology used for blood culture system, the study design, geographical differences, nature of patient population, epidemiological difference of etiological agents and differences in the policies for infection control (15-17).

In our study Gram positive and Gram negative bacteria constituted $61.86 \%$ and $38.14 \%$ respectively. This finding was in contrast with other studies $(12,18-23)$ where Gram negative organisms have taken over Gram positive organisms.

In the present study, the predominant Gramnegative isolates were Escherichia coli (35.14\%) followed by Pseudomonas species $(18.92 \%)$ which was in concordance with other studies (24-28). In contrast to this finding, a study from Mumbai revealed that, Pseudomonas species was the most common cause $(30.37 \%)$ and Escherichia coli amounted upto $16.06 \%$ (22).

In a study from Pakistan to evaluate drug resistance amongst bacterial isolates Escherichia coli was found to be the predominant organism among
Enterobacteriaceae and $P$. aeruginosa and Acinetobacter among non-Enterobacteriaceae group. (25) In a recent study from Lebanon Escherichia coli represented $39.5 \%$ which was very similar to the findings of our study. (24)

In our study predominant Gram positive isolate was Methicillin Resistant Staphylococcus aureus (38.33\%) followed by methicillin sensitive Staphylococcus aureus $(31.67 \%)$, coagulase negative Staphylococci (26.67\%). This finding is similar to other studies where Staphylococcus aureus was the most common isolate $(22,31,32)$. This is in contrast with other studies where coagulase negative Staphylococci has contributed to the blood stream infections in cancer patients. $(27,29,30)$

Among Klebsiella and Escherichia coli meropenem sensitivity was seen in $100 \%$ and $92 \%$ respectively and colistin sensitivity was $75 \%$ and $100 \%$ respectively. Sensitivity to piperacillin + tazobactam was $50 \%$ and $15 \%$ respectively. The susceptibility pattern of Klebsiella was similar to a study from Mumbai where susceptibility to betalactam/beta lactam inhibitors was $56.5 \%$ whereas that of Escherichia coli was in contrast to the same. (33)

A high degree of resistance to cephalosporins among Enterobacteriaceae in our study might be because of the fact that cephalosporins are one of the most commonly used antibiotics for inpatients and for outpatients in developing countries and also because of high incidence self medications as these are available at the counter.(34).

Among non-fermenters, Pseudomonas showed highest sensitivity to polymyxin $b$ and colistin. Acinetobacter species showed highest sensitivity to polymyxin $\mathrm{b}$ and colistin. Sensitivity to meropenem was $100 \%$ 
in Pseudomonas species and $83 \%$ in Acinetobacter species. All our isolates were sensitive to colistin. This was similar to a study from Mumbai where imipenem sensitivity was $91.82 \%$. Colistin showed 94.55\% sensitivity (22) (Fig. 1-9).

Fig.1 Sensitivity pattern of Acinetobacter

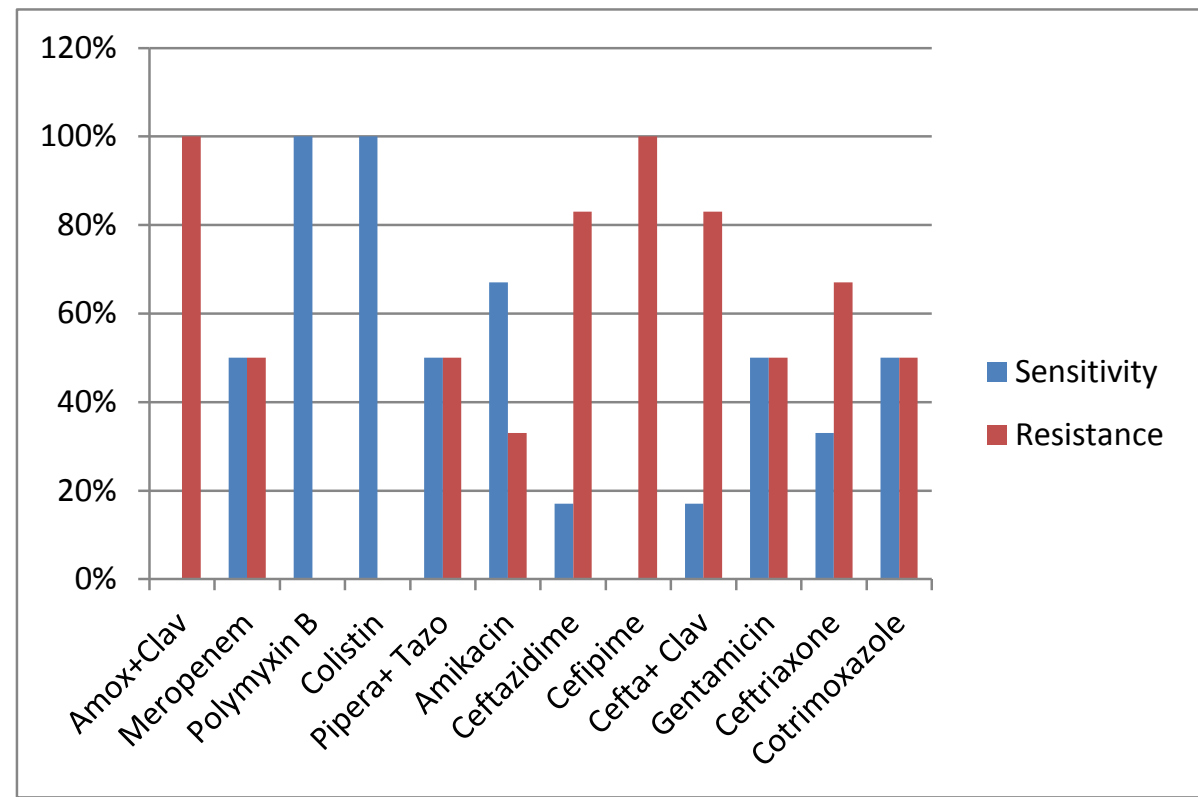

Fig.2 Sensitivity pattern of MRSA

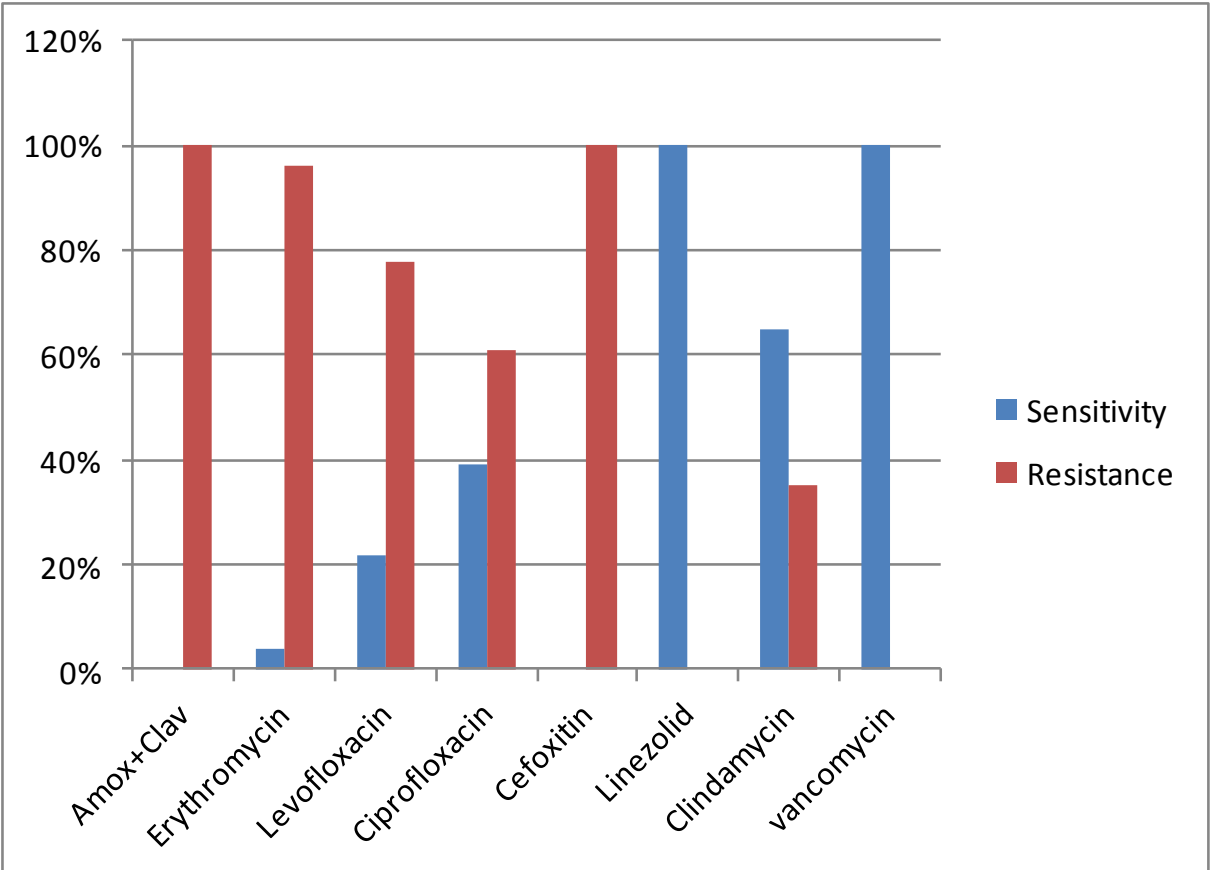


Fig.3 Sensitivity pattern of MSSA

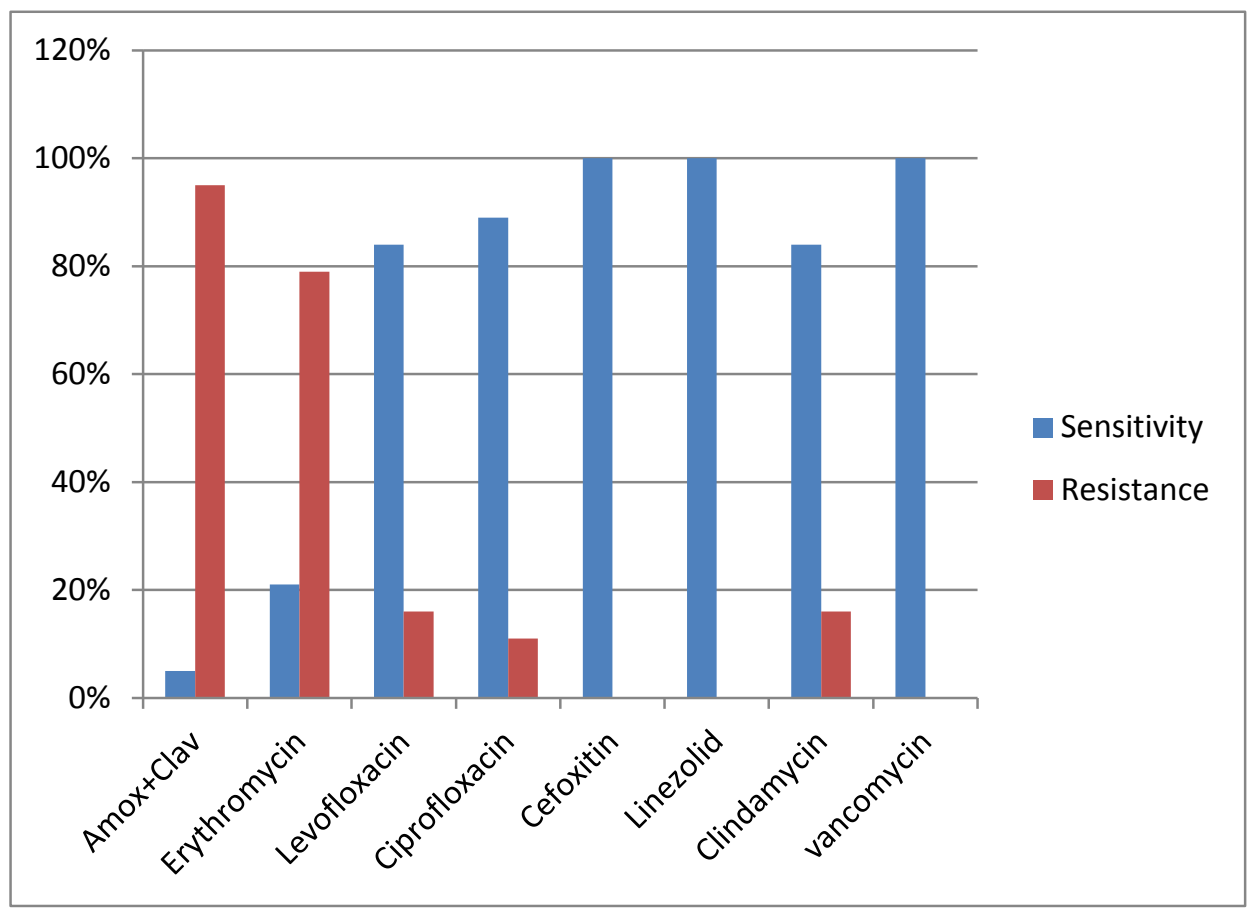

Fig.4 Sensitivity pattern of CONS

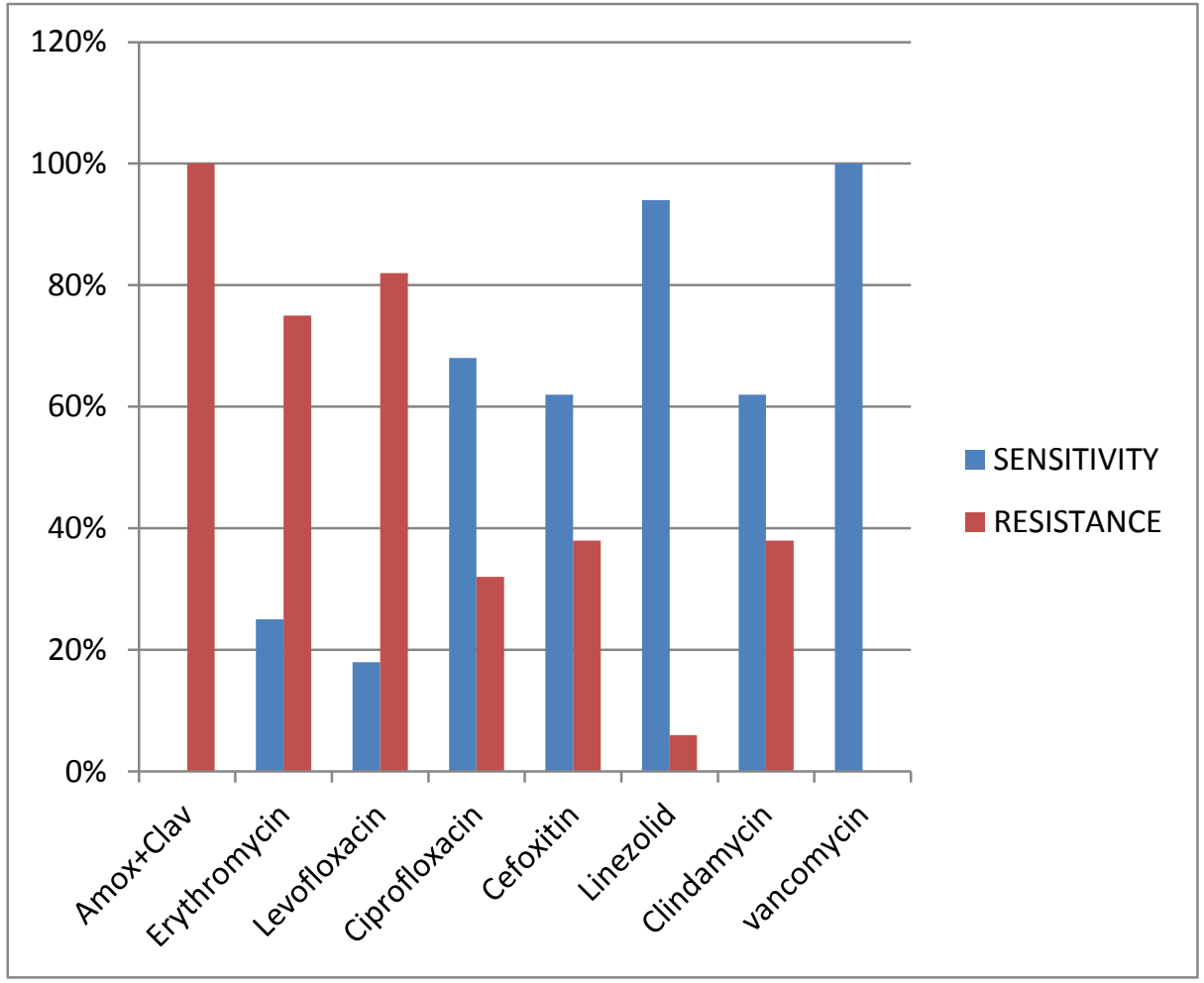


Fig.5 Sensitivity pattern of Escherichia coli

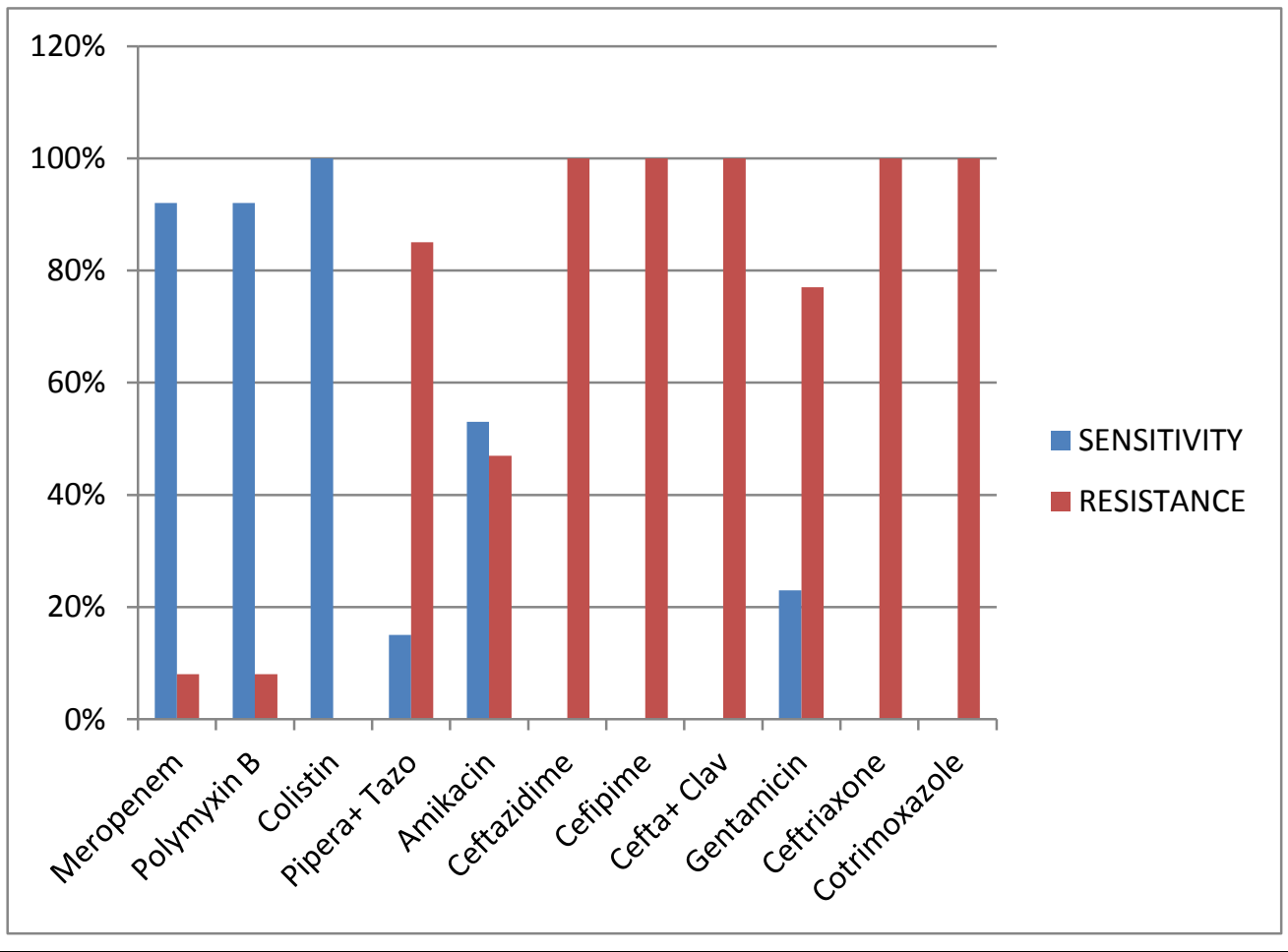

Fig.6 Sensitivity pattern of Pseudomonas

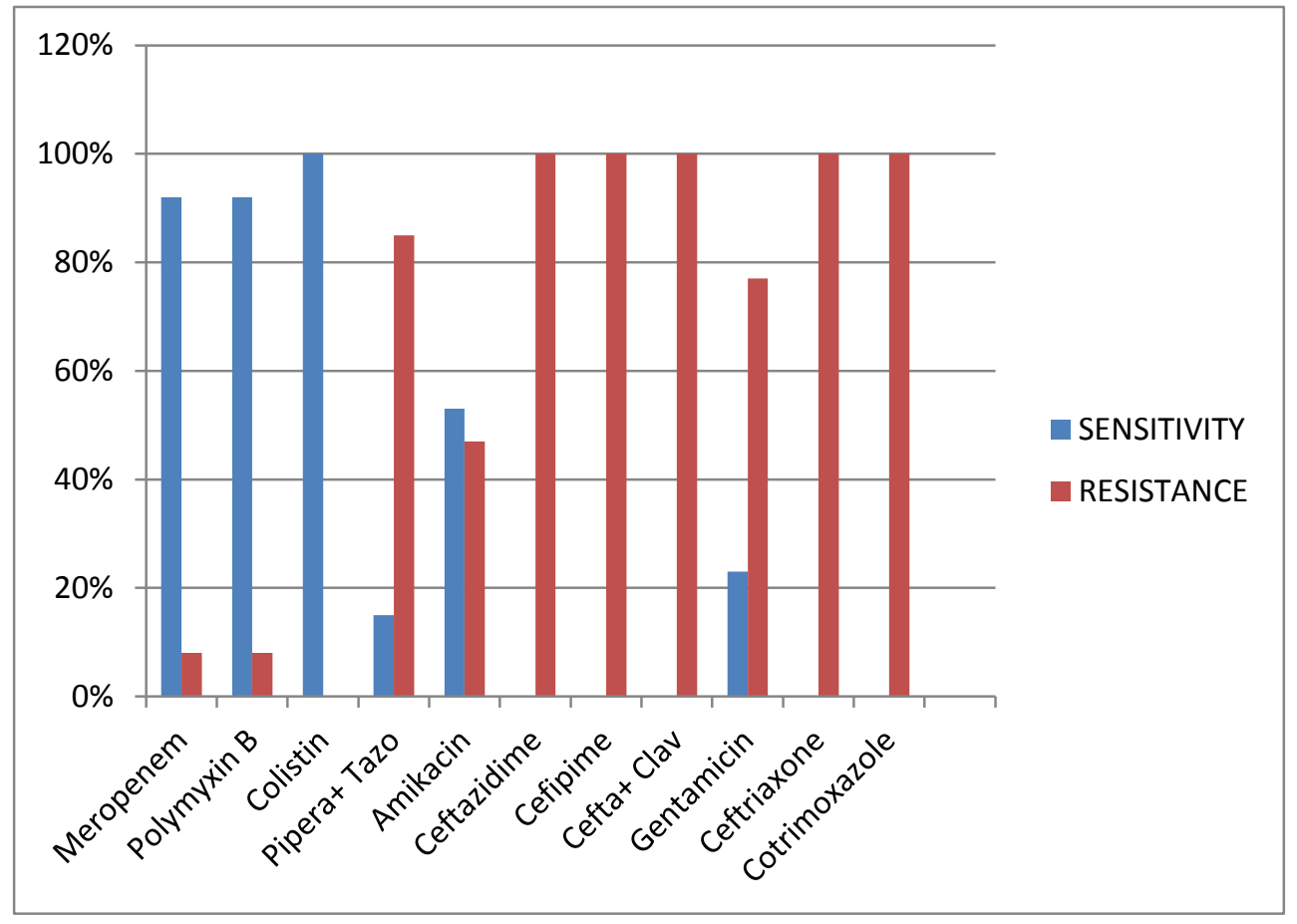


Fig.7.Sensitivity pattern of non-fermenters (other than Pseudomonas)

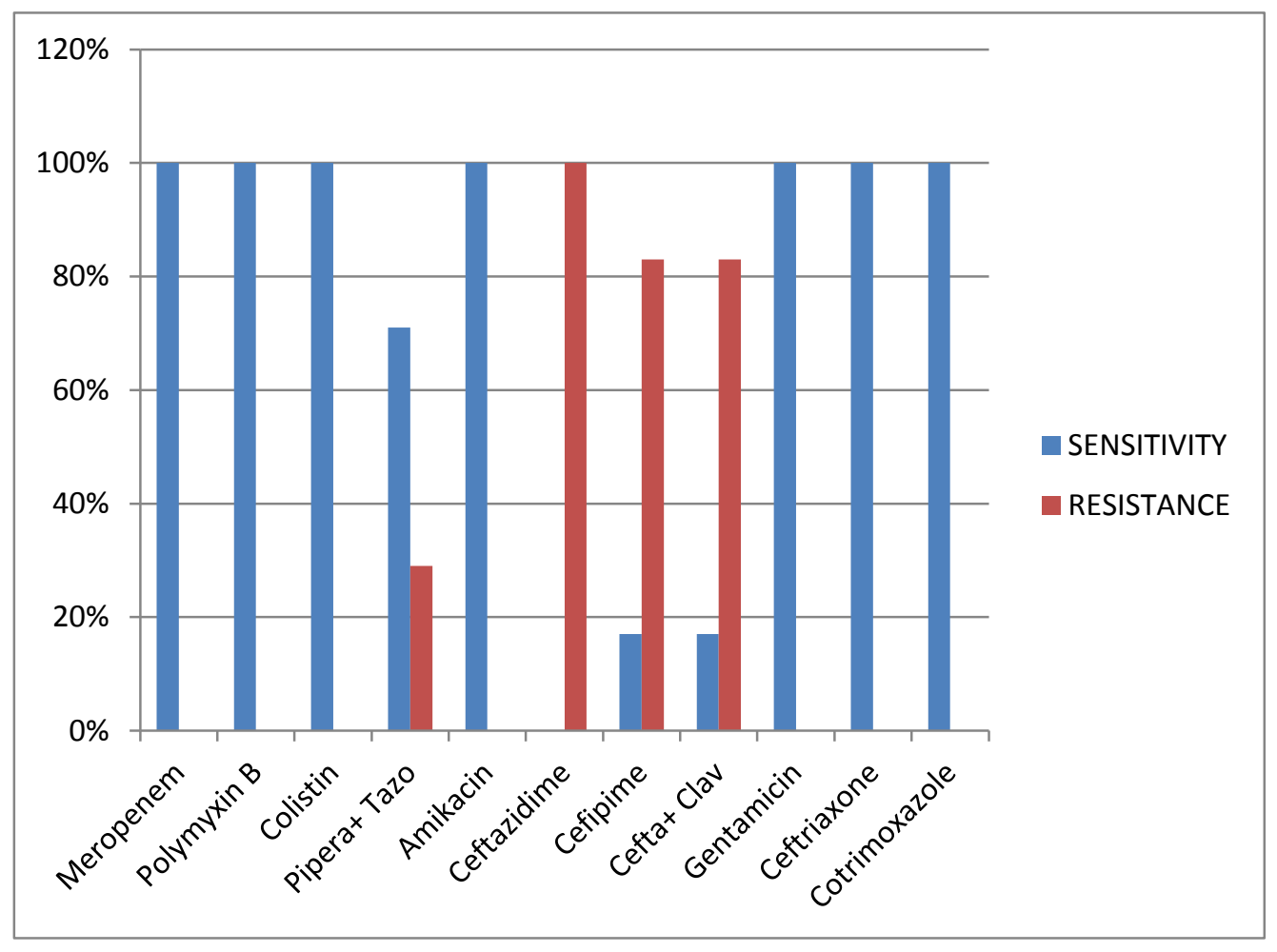

Fig.8 Sensitivity pattern of Klebsiella

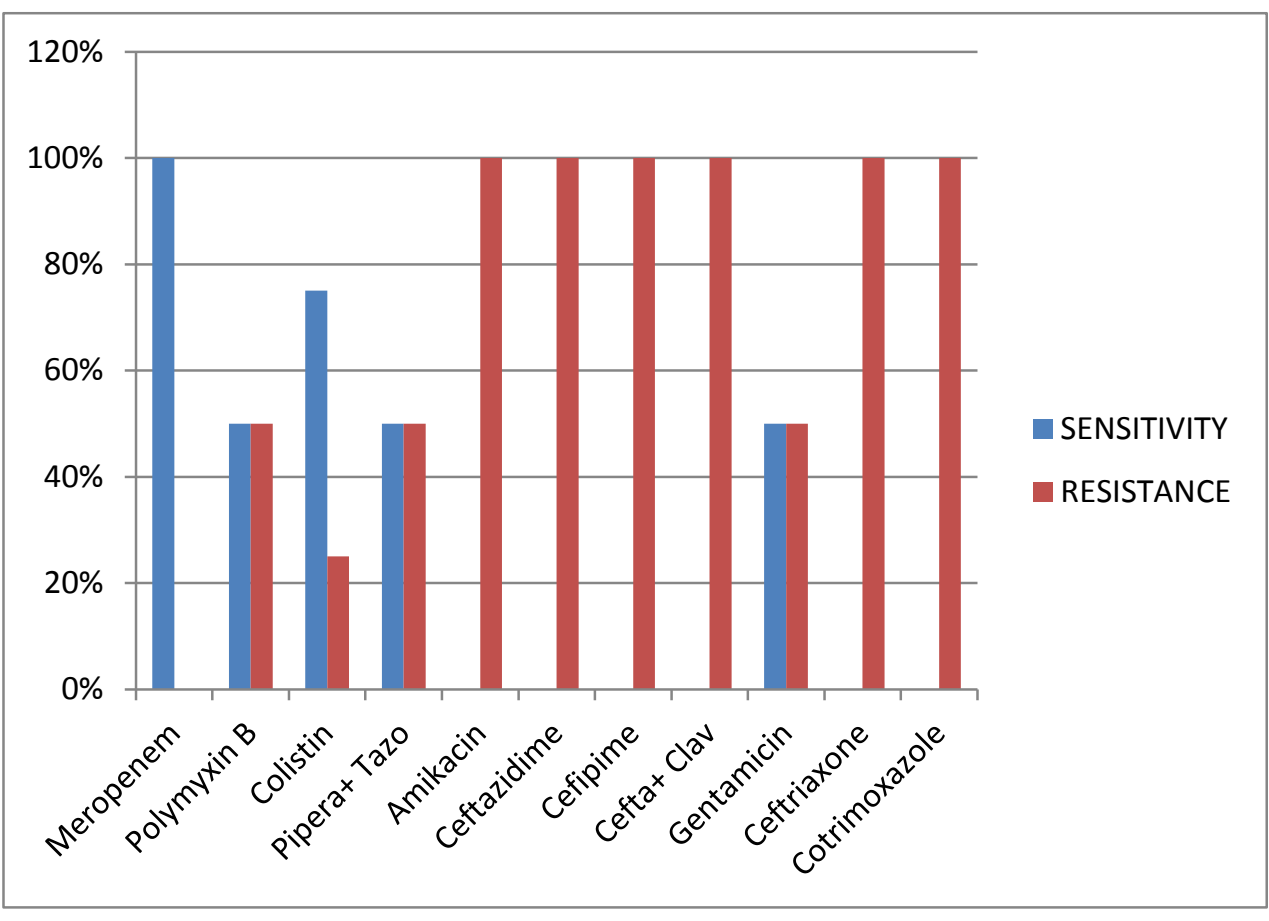


Fig.9 Sensitivity pattern of Enterococcus

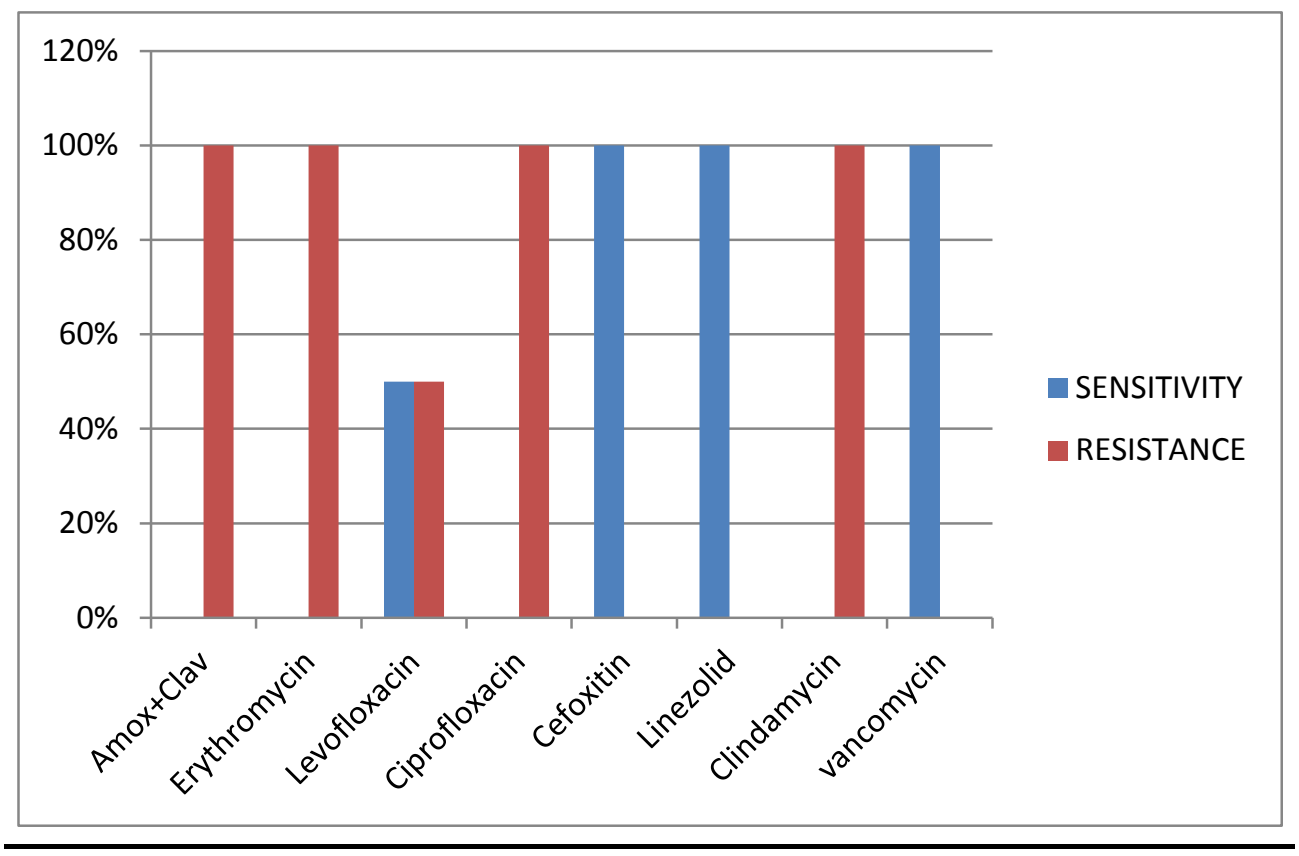

In conclusion, the timely detection of Blood stream infection followed by expeditious identification of pathogen and determination of susceptibility to antimicrobial agents can have great diagnostic and prognostic importance in order to decrease related mortality and morbidity. This will reduce complication and shorten hospital stay and will result in major financial saving for the Institution as well as improving patient care.

\section{References}

1. Diekema DJ, Beekman SE, Chapin KC, Morel KA, Munson E and Doern GV. Epidemiology and outcome of nosocomial and community onset bloodstream infection. J Clin Microbiol 2003;41:3655-60;

http://dx.doi.org/10.1128/JCM.41.8.3655 $-3660.2003$

2. Nucci, Marcio, et al., "Risk factors for death among cancer patients with fungemia." Clinical Infectious Diseases Vol. 27, No. 1, 1998, pp. 107-11
3. Collin, Berjan A., et al., "Evolution, incidence, and susceptibility of bacterial bloodstream isolates from 519 bone marrow transplant patients." Clinical Infectious Diseases, Vol. 33, No. 7, 2001, pp. $947-53$

4. Krcmery, V., et al., "Bacteremia due to multiresistant gram-negative bacilli in neutropenic cancer patients: a case controlled study." Journal of Chemotherapy, Vol. 10, No. 4, 1998, pp. 320-25.

5. Ehni, William F., L. Barth Reller, and Richard T. Ellison III. "Bacteremia in granulocytopenic patients in a tertiarycare general hospital." Reviews of Infectious Diseases Vol. 13, No. 4, 1991, pp. 613-19.

6. Krupova, I., et al., "Bacteremia and fungemia in pediatric versus adult cancer patients after chemotherapy: comparison of etiology, risk factors and outcome." Journal of Chemotherapy Vol. 10, No. 3, 1998, pp. 236-42.

7. Vasudeva, Nikita, Prem Singh Nirwan, and Preeti Shrivastava. "Bloodstream 
infections and antimicrobial sensitivity patterns in a tertiary care hospital of India." Therapeutic Advances in Infectious Disease Vol. 3, No. 5, 2016, pp. 119-27

8. Wasihun, Araya Gebreyesus, et al., "Bacteriological profile and antimicrobial susceptibility patterns of blood culture isolates among febrile patients in Mekelle Hospital, Northern Ethiopia." Springer Plus Vol. 4, No. 1, 2015, p. 314

9. Radha Rani, et al., international Journal of Medical Research and Health sciences, 2017, 6(12): 19-26

10. Mehta, Manjula, Priya Dutta, and Varsha Gupta. "Antimicrobial susceptibility pattern of blood isolates from a teaching hospital in North India." Japanese Journal of Infectious Diseases Vol. 58, No. 3, 2005, p. 174.

11. Arora, Usha, and Pushpa Devi. "Bacterial profile of blood stream infections and antibiotic resistance pattern of isolates." $\mathrm{J}$ K Science Vol. 9, No. 4, 2007, pp. 18690.

12. Shalini S, Kranthi K, Gopalkrishna BK. "The microbiological profile of nosocomial infections in the intensive care unit." Journal of Clinical and Diagnostic Research Vol. 4, 2010, pp. 3109-12.

13. Ghadiri, Hamed, et al., "The antibiotic resistance profiles of bacterial strains isolated from patients with hospitalacquired bloodstream and urinary tract infections." Critical Care Research and Practice 2012, 2012

14. Gohel, Kalpesh, et al., "Bacteriological profile and drug resistance patterns of blood culture isolates in a tertiary care nephrourology teaching institute." BioMed Research International2014, 2014.

15. Zenebe, Tizazu, et al., "Invasive bacterial pathogens and their antibiotic susceptibility patterns in Jimma
University specialized hospital, Jimma, Southwest Ethiopia." Ethiopian Journal of Health SciencesVol. 21, No. 1, 2011, pp. 1-8.

16. Dagnew, Mulat, et al., "Bacterial profile and antimicrobial susceptibility pattern in septicemia suspected patients attending Gondar University Hospital, Northwest Ethiopia.'BMC Research Notes Vol. 6, 2013

17. Vanitha, Rani N., et al., "A retrospective study on blood stream infections and antibiotic susceptibility patterns in a tertiary care teaching hospital."Vol. 4, No. 1, 2012, pp. 543-48.

18. Gupta, Shilpi, and Bineeta Kashyap. "Bacteriological profile and antibiogram of blood culture isolates from a tertiary care hospital of North India." Tropical Journal of Medical Research Vol. 19, No. 2, 2016, p. 94.

19. Pal, Nidhi, and RaMaMuRthy Sujatha. "Antimicrobial resistant pattern of blood culture isolates, among septicaemia suspected patients." National Journal of Laboratory Medicine Vol 5, No. 1, 2016, pp. 17-21.

20. Sonawane, Jyoti P., et al., "Bacteriological profile and antimicrobial susceptibility of blood culture isolates from tertiary care hospital, Navi Mumbai." Journal of Medical Science and Clinical Research Vol. 5, No. 1, 2016, pp. 17-21.

21. Prabhash, Kumar, et al., "Blood stream infections in cancer patients: A single center experience of isolates and sensitivity pattern." Indian Journal of CancerVol. 47, No. 2, 2010, p. 184.

22. Chen, Chien-Yuan, et al., "Trends and antimicrobial resistance of pathogens causing bloodstream infections among febrile neutropenic adults with hematological malignancy." Journal of the Formosan Medical Association Vol 103, No. 7, 2004, pp. 526-32 
23. Moghnieh, Rima, et al., "Third generation cephalosporin resistant Enterobacteriaceae and multidrug resistant gram-negative bacteria causing bacteremia in febrile neutropenia adult cancer patients in Lebanon, broad spectrum antibiotics use as a major risk factor, and correlation with poor prognosis." Frontiers in Cellular and Infection Microbiology Vol. 5, 2015.

24. Irfan, Seema, et al., "Emergence of Carbapenem resistant Gram negative and vancomycin resistant Gram-positive organisms in bacteremic isolates of febrile neutropenic patients: a descriptive study." BMC Infectious Diseases, Vol. 8, No. 1, 2008, p. 80.

25. Al-Otaibi, Fawzia E., et al., "Prevalence and risk factors of Gram-negative bacilli causing blood stream infection in patients with malignancy." Saudi Medical JournalVol. 37, No. 9, 2016, p. 979.

26. Gustinetti, Giulia, and Malgorzata Mikulska. "Bloodstream infections in neutropenic cancer patients: a practical update." Virulence Vol. 7, No. 3, 2016, pp. 280-97.

27. Bansal, S, and SH Advani. "Pattern of bloodstream infections in patients with hematological malignancies in a tertiary care centre." Indian Journal of Cancer Vol. 51, No. 4, 2014, pp. 447-449.

28. Ahmed, N.H., F.K. Baruah, and R.K. Grover. "Letter to Editor: Staphylococcal Blood Stream Infections in Cancer Patients." Annals of Medical and Health
Sciences Research Vol. 5, No. 3, 2015, pp. 226-27.

29. Velasco, Eduardo, et al., "Epidemiology of bloodstream infections at a cancer center." Sao Paulo Medical Journal Vol. 118, No. 5, 2000, pp. 131-38.

30. Babu, K. Govind, et al., "Bloodstream infections in febrile neutropenic patients at a tertiary cancer institute in South India: A timeline of clinical and microbial trends through the years." Indian Journal of Medical and Paediatric Oncology: Official Journal of Indian Society of Medical \& Paediatric Oncology Vol. 37, No. 3, 2016, p. 174.

31. Kang, Cheol-In, et al., "Bloodstream infections in adult patients with cancer: clinical features and pathogenic significance of Staphylococcus aureus bacteremia." Supportive Care in Cancer Vol. 20, No. 10, 2012, pp. 2371-78

32. Singhal, T., S. Shah, and R. Naik. "The microbial etiology and antimicrobial susceptibility of bloodstream infections in patients with cancer at a private tertiary care hospital in Mumbai, India." Indian Journal of Cancer Vol. 53, No. 3, 2016, p. 452.

33. Nathisuwan, Surakit, David S. Burgess, and James S. Lewis. "Extended-spectrum $\beta$-lactamases: epidemiology, detection, and treatment." Pharmacotherapy: The Journal of Human Pharmacology and Drug Therapy, Vol. 21, No. 8, 2001, pp. 920-28.

\section{How to cite this article:}

Mukta Sawargaonkar, Nazneen Siddiqui, Joson Mathew and Arvind Gaikwad. 2019. Bacteriological Profile of Blood Stream Infections Along with their Antibiogram at Government Cancer Hospital, Aurangabad. Int.J.Curr.Microbiol.App.Sci. 8(05): 2082-2091. doi: https://doi.org/10.20546/ijcmas.2019.805.243 